\title{
Analytical study of gyrophase, pitch angle and normal speed of particles leaving an ideal magnetohydrodynamic discontinuity surface*
}

\author{
William P. Wilkinson \\ Division of Mathematical Sciences, \\ University of Brighton, Lewes Road, Brighton BN2 4GJ, UK \\ e-mail:W.P.Wilkinson@brighton.ac.uk
}

October 8, 2020

\begin{abstract}
Particle populations that have velocity distributions with only a small spread of gyrophase angles are commonly observed in the vicinity of magnetohydrodynamic (MHD) discontinuity surfaces such as collisionless shocks. Previous theoretical particle trajectory studies have concentrated on ion behavior at an ideal planar Earth's bow shock and have either assumed that a gyrotropic incident initial velocity distribution is reflected at the surface or instead focused on unique fixed initial gyrophase and pitch angle values specified by the generation mechanism assumed for the particle. In this analytical study of trajectories of particles departing an ideal planar MHD surface we demonstrate that a particle's initial gyrophase and pitch angle determine completely whether it will escape the surface or return to it, regardless of its initial energy. We identify the region in initial gyrophase-pitch angle space which leads to trajectories that return to the surface of the discontinuity. The speed normal to the surface of a returning particle, which can affect its ability to traverse the discontinuity, is shown to increase or decrease compared to its initial value
\end{abstract}

*This article was published on 24 August 2020 in Phys. Rev. E 102, 023211 
according only to the orientation of its guiding-center motion in the frame of reference in which the discontinuity is at rest and the incoming plasma flow is aligned with the constant magnetic field. The dependence of our results on the direction of the upstream magnetic field is illustrated. Our general analytical results are discussed in the context of observations at the Earth's bow shock.

\section{Introduction}

The theory of Magnetohydrodynamics (MHD) predicts the existence of several types of discontinuities in plasma flows. These are classified as shocks, rotational, tangential or contact discontinuites according to whether or not the fluid crosses the boundary and to whether or not the magnetic field has a component normal to the surface of the discontinuity (e.g. $[4,37])$. The large-scale properties of boundaries observed in space plasmas, such as the Earth's magnetopause or bow shock, are consistent with those of one or other of the above MHD discontinuities (e.g., [37]) and a wide variety of plasma phenomena observed in space are therefore described and modelled within the theoretical framework of MHD. Such is the case, to name but a few, of tangential and rotational discontinuity-driven foreshock bubbles [26], the propagation of waves along contact discontinuities in the solar atmosphere [38], the interaction of a magnetosheath jet with a magnetopause modelled as a tangential discontinuity [43], or the effect of interplanetary tangential discontinuities on the magnetosheath and the magnetopause [19].

Boundaries separating different plasma states are often the site of diverse particle populations that play important roles in the local transport of energy and which are therefore the subject of intense research. In this paper we study how the pitch angle and the gyrophase angle of a particle departing the surface of an ideal planar MHD discontinuity affect whether it will return to the surface or escape. The motivation for this study came from the high-resolution measurements taken by the CLUSTER spacecraft showing that two distinct ion populations observed at Earth's bow shock, namely, the so-called "reflected-gyrating" ions and the "field-aligned beams" appear to emerge from a single gyrophase-bunched population of solar wind ions reflected at the shock ramp $[34,25]$. The properties of these two populations are reviewed in [5] and are summarized here. The reflected-gyrating ions are known to play a key part in the collisionless heating of the solar 
wind by Earth's shock under typical high Mach number conditions (e.g., $[40,41,2])$. These ions depart the shock surface with a restricted range of gyrophase angles, i.e., are "gyrophase-bunched" ([21]). Then, depending on the orientation of the interplanetary magnetic field, they may return to the shock surface with a sufficiently high normal speed to overcome the electrostatic potential jump at the shock ramp and pass downstream into the magnetosheath (e.g. see review articles by $[18,2]$ ). Observations at the bow shock confirm that the velocity distributions immediately downstream, which consist of those solar wind ions directly transmitted through the shock and the reflected-gyrating ions, have a combined effective temperature consistent with the Rankine-Hugoniot conservation relations [40]. At this stage the plasma is non-gyrotropic but it becomes thermalized further downstream $[40,7,41]$. Studies of the thermalization process have focussed on ion cyclotron and mirror instabilities and assumed bi-Maxwellian velocity distributions with anisotropic temperatures (e.g., [13]). However, the nongyrotropic and spatially inhomogeneous properties of the distributions are likely to affect the microphysics of the thermalization (e.g., $[3,8]$ ).

Field-aligned beams receive their name from the observation that they propagate upstream away from the shock at small angles to the interplanetary magnetic field (see the review articles by e.g., [42, 5]). These ion beams are the most important source of free energy in the foreshock region [29]. They can experience disruption by a beam-plasma instability (e.g., $[22,11,12])$ leading to gyrophase-bunched distributions observed in association with high-amplitude quasi-monochromatic MHD-like waves over a wide range of distances from the Earth's bow shock [30, 28, 33, 31]. A process of pitch angle scattering by low frequency waves has been suggested as a possible explanation for how the beam ions are initially selected out of the reflected-gyrating non-gyrotropic distribution at the shock ramp [34, 25]. Other intriguing and as yet unexplained features of the beams are their significant lack of $\mathrm{He}^{++}$compared to the incident solar wind $[23,10]$, the occasional appearence of high-energy tails of high pitch angle in their velocity distributions [32] and striking differences between how the parallel and perpendicular thermal speeds depend on the geometry of the shock [29].

The above observations have been discussed in some detail because they are examples of how gyrophase and pitch angle structure are important features of particle populations in the vicinity of MHD discontinuities. Test particle simulations confirm this point (e.g., [6]). A full understanding of the micro-physics of the processes taking place at boundaries in space plasmas 
often requires plasma kinetic theory (e.g., [1]). Nevertheless, key insights into fundamental phenomena at MHD discontinuity surfaces have been gained by analyzing the behaviour of individual particle trajectories in a steady-state approximation of the electromagnetic fields surrounding the discontinuity (e.g., $[6,14,36,15])$. This well-established approach is the one we will adopt in the current study, in an attempt to understand better the part played by initial gyrophase and pitch angle in the final destination of a particle leaving the surface of the discontinuity. Previous theoretical analysis of gyrophase bunching has focused on the evolution of velocity distributions assumed to be initially gyrotropic [20]. By contrast, our aim here is to identify which pitch angle and gyrophase angle values at the moment that a particle departs the surface of an ideal MHD discontinuity lead to the particle's trajectory reencountering the surface as opposed to escaping upstream. We also examine the effect of pitch angle and gyrophase angle on a particle's normal speed at re-encounter. This is an important parameter in the context of collisionless shocks as it affects the ability of an ion to overcome the electrostatic potential jump at the shock ramp.

\section{Theoretical Foundations}

The analysis of particle trajectories at an infinite plane surface in a collisionless, homogeneous and steady plasma flow is particularly straightforward when performed in the Hoffman-Teller (HT) frame of reference [9, 39, 4]. This frame moves along the surface with the precise velocity that makes the incident bulk flow align with the upstream magnetic field $\mathbf{B}$, which is assumed to be constant. As there is no motional electric field in the HT frame a particle's trajectory is reduced to the combination of a guiding-centre motion along the magnetic field direction and a gyrational motion around it. This simplified trajectory can be convenientely described in the coordinate system introduced by [39] and depicted in Figure 1. It consists of three orthogonal unit vectors $\hat{\mathbf{b}}, \hat{\zeta}, \hat{\xi}$, with $\hat{\mathbf{b}}$ a unit vector pointing upstream along the direction of the magnetic field $\mathbf{B}$ and $\hat{\zeta}, \hat{\xi}$ defined by $\hat{\xi}=(\hat{\mathbf{b}} \times \hat{\mathbf{n}}) /$ $|\hat{\mathbf{b}} \times \hat{\mathbf{n}}|$ and $\hat{\zeta}=\hat{\xi} \times \hat{\mathbf{b}}$. Here the unit vector $\hat{\mathbf{n}}$ points upstream along the direction normal to the planar surface. In this system (refer to Figure 1), the velocity after time $t$ of a particle leaving the upstream side of the surface at $t=0$ with constant guiding centre velocity $\mathbf{v}_{b}=v_{b} \hat{\mathbf{b}}$ and (non-negative) 


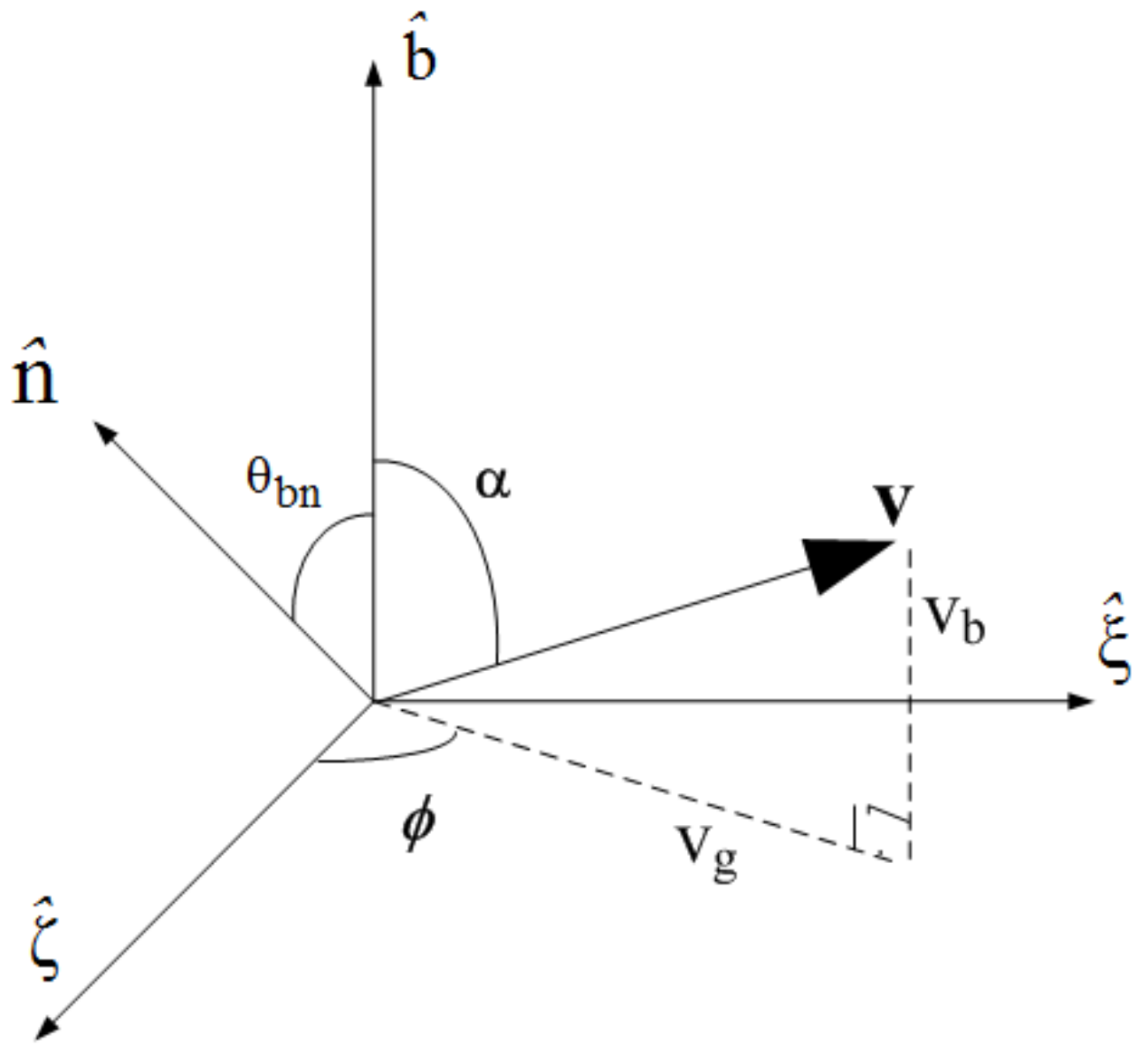

Figure 1: Orthogonal coordinate axes used for the description of particle trajectories (adapted from [39], fig. 2). The unit vectors $\hat{\mathbf{b}}$ and $\hat{\mathbf{n}}$ point upstream along the magnetic field and surface normal directions, respectively. The particle's velocity is $\mathbf{v}$, its pitch angle is $\alpha$ and gyrophase is $\phi$. Particle gyromotion takes place in the $\hat{\zeta}-\hat{\xi}$ plane. 
constant gyrational speed $v_{g}$, is given by (cf. equation (6) in [39]):

$$
\mathbf{v}(t)=v_{b} \hat{\mathbf{b}}+v_{g}[\hat{\zeta} \cos \phi(t) \pm \hat{\xi} \sin \phi(t)]
$$

where $\phi(t)=\Omega t+\phi_{0}$ is the particle's time-dependent gyrophase expressed as a function of its gyrofrequency $\Omega$ and initial gyrophase $\phi_{0}$. The gyrophase angle is arbitrarily measured from the $\hat{\zeta}$ axis and is positive in an anticlockwise sense (see Figure 1) for a negatively charged particle when $\mathbf{B}=|\mathbf{B}| \hat{\mathbf{b}}$ or for a positive ion when $\mathbf{B}=-|\mathbf{B}| \hat{\mathbf{b}}$ (both of these correspond to the plus sign in front of the $\hat{\xi}$ term in equation (1)). Conversely, $\phi(t)$ increases in a clockwise sense for a positive ion when $\mathbf{B}=|\mathbf{B}| \hat{\mathbf{b}}$ or a negatively charged particle when $\mathbf{B}=-|\mathbf{B}| \hat{\mathbf{b}}$ (minus sign in front of $\hat{\xi}$ in equation (1)). We note that $v_{b}>0$ corresponds to a guiding-centre motion directed upstream (i.e. $\mathbf{v}_{b}$ has a component along $+\hat{\mathbf{b}}$ ). The particle's pitch angle, which lies in the range $\left[0^{\circ}, 180^{\circ}\right]$ is given by (Figure 1 ):

$$
\alpha=\cos ^{-1} \frac{v_{b}}{\sqrt{v_{b}^{2}+v_{g}^{2}}}
$$

Integrating equation (1) with respect to time leads to the particle's displacement (cf. equation (7) in [39]):

$$
\mathbf{r}(t)=v_{b} t \hat{\mathbf{b}}+\frac{v_{g}}{\Omega}\left[\hat{\zeta}\left(\sin (\phi(t))-\sin \phi_{0}\right) \pm \hat{\xi}\left(\cos \phi_{0}-\cos (\phi(t))\right)\right]
$$

In this article we aim to determine analytically the sets of initial gyrophase and pitch angles which lead to trajectories that return to the surface of the discontinuity and those which lead to particles escaping the surface without re-encountering it. We also wish to establish how the magnitude of a particle's speed normal to the surface for a returning particle might be related to its initial velocity at the moment of departing. To help address these questions we obtain the particle's speed and displacement as a function of time in the direction normal to the surface from the dot product of (1) and (2), respectively, with $\hat{\mathbf{n}}$ (cf. equations (18) and (19) in [39]):

$$
\begin{gathered}
v_{n}(t)=v_{b} \cos \theta_{b n}+v_{g} \sin \theta_{b n} \cos \left(\Omega t+\phi_{0}\right) \\
r_{n}(t)=v_{b} t \cos \theta_{b n}+\frac{v_{g}}{\Omega} \sin \theta_{b n}\left[\sin \left(\Omega t+\phi_{0}\right)-\sin \phi_{0}\right]
\end{gathered}
$$

where $\theta_{b n}$ is the acute angle between $\hat{\mathbf{b}}$ and $\hat{\mathbf{n}}$ (see Figure 1 ). In what follows our focus will be on trajectories leaving the MHD discontinuity surface on its upstream side but the analysis can be applied to trajectories downstream. 


\section{Initial Gyrophase and Pitch Angle of Re- turning and Escaping Particles}

In this section we show that a particle's pitch angle in the HT reference frame and its initial gyrophase uniquely determine whether it will return to the surface of an ideal MHD discontinuity or escape upstream.

A particle leaving the surface on the upstream side must have an initial speed in the direction of $+\hat{\mathbf{n}}$, i.e. (cf. equation $(3)$ ):

$$
v_{n}(0)=v_{b} \cos \theta_{b n}+v_{g} \sin \theta_{b n} \cos \phi_{0}>0
$$

Dividing by the positive quantity $v_{g} \sin \theta_{b n}$ and rearranging, it follows that:

$$
\cos \phi_{0}>-\frac{v_{b} \cos \theta_{b n}}{v_{g} \sin \theta_{b n}}
$$

This condition on a particle's initial gyrophase $\phi_{0}$ is particularly restrictive on particles with $v_{b}<0$ (guiding centre velocities pointing downstream). In terms of the particle's pitch angle $\alpha$ in the HT frame, (see Figure 1) condition (6) is equivalent to:

$$
\cos \phi_{0}>-\cot \alpha \cot \theta_{b n}
$$

The distance from the surface as a function of time of particles satisfying (7) (i.e. departing the surface towards upstream) can be calculated using (4). Figure 2 displays the combinations of $\left(\alpha, \phi_{0}\right)$ which lead to a particle either returning to the surface (labelled $\mathrm{A}$ on the diagram) or escaping upstream (labelled B and C). Results are included for three values of $\theta_{b n}$. Pitch angles $\alpha$ lower than $90^{\circ}$ (below the horizontal dashed line in the panels) correspond to upstream-directed guiding centre motion $\left(v_{b}>0\right)$. The figure reveals that particles returning to the surface (region A) originate from a very restricted part of gyrophase-pitch angle space. Those with downstream-directed $\mathbf{v}_{b}$ $\left(90^{\circ}<\alpha<180^{\circ}\right.$, i.e. above the horizontal dashed line) only have gyrophase angles in the first and fourth quadrants, where $\cos \phi_{0}>0$, as expected from (7).

The complete absence of returning particles (region A) with $180^{\circ}<$ $\phi_{0}<270^{\circ}$ in Figure 2 can be explained as follows. At the time $t=t^{*}$ of re-encounter with the surface, the normal velocity of a particle must be downstream-pointing $\left(v_{n}\left(t^{*}\right)<0\right)$, i.e. (cf. (3)): 

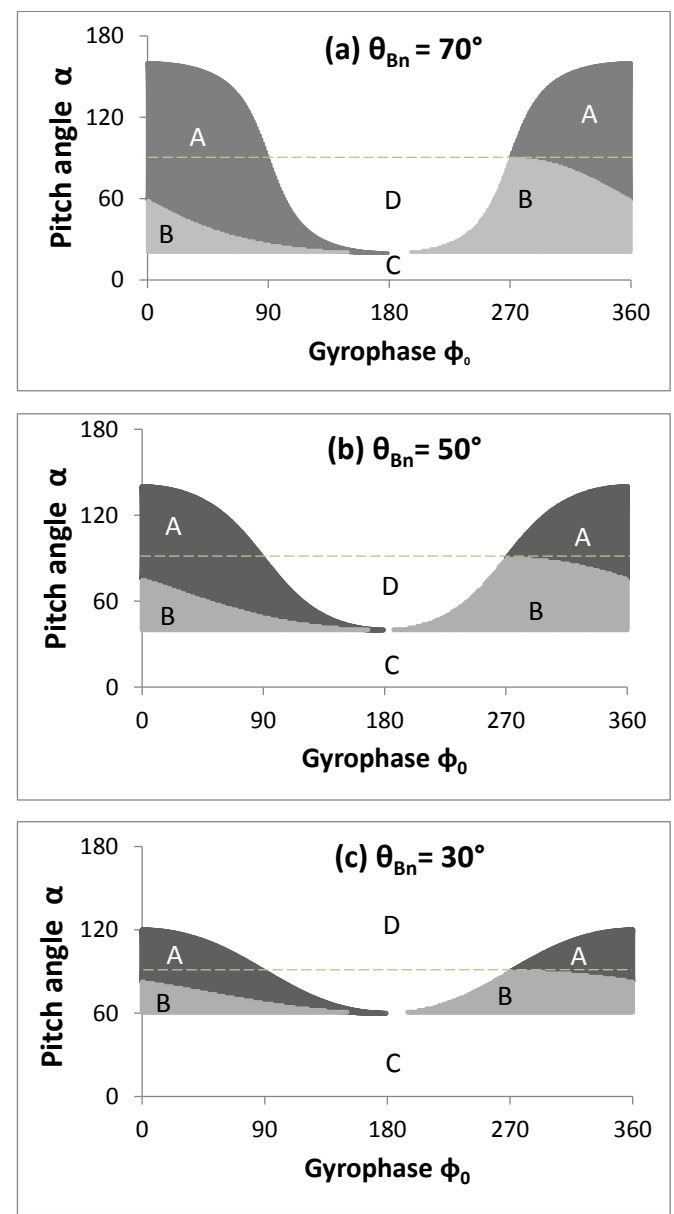

Figure 2: Possible initial gyrophase and pitch angles in the HT frame of particles re-encountering the surface (regions labelled A in the panels), escaping upstream after re-approaching but not re-encountering the surface (regions labelled B), and escaping without ever turning back towards the surface (labelled C). Region D corresponds to particles with initial velocities pointing downstream. The horizontal dashed line at $\alpha=90^{\circ}$ marks the boundary between particles whose guiding-centre motion is directed downstream $\left(\alpha>90^{\circ}\right)$ and those with guiding-centre motions pointing upstream. The top, middle, and bottom panels ${ }^{8}$ correspond to $\theta_{b n}=70^{\circ}, 50^{\circ}$ and $30^{\circ}$, respectively. 


$$
\cos \left(\Omega t^{*}+\phi_{0}\right)<-\cot \alpha \cot \theta_{b n}
$$

In addition, as the particle's normal displacement at $t=t^{*}$ is zero, (4) implies that,

$$
\frac{v_{b} \cos \theta_{b n}}{v_{g} \sin \theta_{b n}} \equiv \cot \alpha \cot \theta_{b n}=\frac{\sin \phi_{0}-\sin \left(\Omega t^{*}+\phi_{0}\right)}{\Omega t^{*}}
$$

Expressions (8) and (9) together with (7) (the requirement that $v_{n}(0)>$ $0)$, imply that:

$$
\cos \phi_{0}>\frac{\sin \left(\Omega t^{*}+\phi_{0}\right)-\sin \phi_{0}}{\Omega t^{*}}>\cos \left(\Omega t^{*}+\phi_{0}\right)
$$

and when $\phi_{0}$ is in quadrant $3\left(\cos \phi_{0}<0, \sin \phi_{0}<0\right)$ there are simply no values of $t^{*}$ for which $\cos \phi_{0}>\cos \left(\Omega t^{*}+\phi_{0}\right)$ and $\sin \phi_{0}>\sin \left(\Omega t^{*}+\phi_{0}\right)$ simultaneously, i.e. particles with $\phi_{0}$ in the range $\left[180^{\circ}, 270^{\circ}\right]$ cannot return to the surface after leaving on its upstream side.

We next consider the trajectories of particles that do not return to the surface of the discontinuity. These can be grouped according to whether the particle turns back towards the surface for a time (labelled B in Figure 2) or, instead, continually keeps getting further away (C). Figure 2 reveals that these two populations occupy distinct areas of gyrophase-pitch angle space. A trajectory can only turn back towards the surface if the particle's normal speed becomes zero some time after departing. This requires that (cf. (3)):

$$
-1 \leq \frac{v_{b} \cos \theta_{b n}}{v_{g} \sin \theta_{b n}} \equiv \cot \alpha \cot \theta_{b n} \leq 1
$$

Conversely, a sufficient condition for a particle to escape upstream without ever approaching the surface again is that $|\cot \alpha|>\tan \theta_{b n}$. For particles with $v_{b}>0$ this corresponds to pitch angles in the range:

$$
0^{\circ}<\alpha<90^{\circ}-\theta_{b n}
$$

This result was derived geometrically for particles with upstream-directed guiding centre speeds by [42]. It explains why ion beams observed in the Earth's foreshock are increasingly field-aligned as $\theta_{b n}$ becomes more perpendicular [42]. The horizontal gyrotropic band of small pitch angles labelled $\mathrm{C}$ on Figure 2 is occupied by trajectories satisfying (12) which never turn back towards the surface of the discontinuity. 
Escaping particles that approach the surface for a while (satisfying (11) and labelled B on Figure 2) arise from specific portions of $\alpha-\phi_{0}$ space, narrower in pitch angle but wider in gyrophase angles than for the returning particles (A). The boundary between populations B and A corresponds to combinations of $\alpha$ and $\phi_{0}$ that result in trajectories tangential to the surface (i.e. for which $\left.v_{n}\left(t^{*}\right)=0\right)$. The region labelled D in Figure 2 corresponds to particles with initial velocities directed downstream (i.e. with $\left.v_{n}(0)<0\right)$. The boundary between region $\mathrm{D}$ and regions $\mathrm{B}$ and $\mathrm{A}$ is the curve $\cos \phi_{0}=$ $-\cot \alpha \cot \theta_{b n}\left(\right.$ or $\left.\alpha=\cot ^{-1}\left(-\cos \phi_{0} \tan \theta_{b n}\right)\right)$, where $v_{n}(0)=0$ (cf. equation (5)). Note that as $\theta_{b n}$ decreases, the height of this curve decreases while band $\mathrm{C}$ becomes wider (cf. equation (12)) and both effects conspire to squeeze the range of pitch angles available to populations A and B (see figure 2).

The reflected-gyrating ions observed immediately upstream of Earth's bow shock are an example of a gyrophase-bunched population, as mentioned in our Introductory section above. Their underlying properties have been explained by assuming that these are a subset of the incident solar wind ions which have been reflected quasi-specularly at the shock (see, for example, the review by [18]). In "cold-particle" trajectory studies (where all incident particles have the same velocity as the bulk of the solar wind) ions that are specularly reflected from an ideal planar shock are predicted to have $\alpha=2 \theta_{b n}$ and $\phi_{0}=0([39])$. These values would place such ions firmly in region $\mathrm{A}$ of Figure 2 for $\theta_{b n}=70^{\circ}$ and $50^{\circ}$ and in the C-B regions boundary for $\theta_{b n}=30^{\circ}$. This is consistent with the finding that cold specularly reflected ions return to the planar shock for $\theta_{b n} \geq 39.9^{\circ}$ ([39]).

\section{Speed Normal to the Surface of Returning Particles}

Solar wind ions with insufficient normal speed to overcome the electrostatic potential jump at Earth's bow shock experience reflection (e.g., [2] and references therein). If these ions are able to return to the shock with increased normal speed after gyration in the upstream magnetic field, they may then be able to overcome the potential jump and pass downstream, contributing to the collisionless thermalization of the incident plasma. This specific example illustrates the relevance to the dynamics of MHD discontinuities of the speed normal to the surface of a returning particle. We shall now demonstrate that 
the direction of a particle's guiding centre velocity in the HT frame (indicated by the sign of $v_{b}$ ) determines whether or not a returning particle will have a speed normal to the surface greater than its initial normal speed.

The time $t=t^{*}$ at which a particle re-encounters the surface can be calculated from equation (9), which we re-write below in a slightly simplified format:

$$
\frac{v_{b}}{v_{g}} \cot \theta_{b n}=\frac{\sin \phi_{0}-\sin \left(\Omega t^{*}+\phi_{0}\right)}{\Omega t^{*}}
$$

Thus, the re-encounter time depends on the ratio $v_{b} / v_{g}$ (which is related to $\alpha$, the particle's pitch angle) and the angles $\phi_{0}$ and $\theta_{b n}$. Alternatively, for any specific $\theta_{b n}$ one may regard the ratio $v_{b} / v_{g}$ that a particle must have in order to re-encounter the surface as being a function of $\phi_{0}$ and of the time $t^{*}$. Note that equation (13) indicates that $v_{b}$ changes sign at $\Omega t_{1}^{*}=$ $(2 n+1) \pi-2 \phi_{0}$ and at $\Omega t_{2}^{*}=2 \pi$, with $n$ the integer that makes $0<\Omega t_{1}^{*}<2 \pi$ ( $n=0,1,1,2$ in quadrants $1,2,3$ and 4 , respectively). We also note that $v_{b} \cot \theta_{b n} / v_{g} \rightarrow-\cos \phi_{0}$ as $\Omega t^{*} \rightarrow 0$, through application of L'Hôpital's Rule in equation (13). These features are illustrated in Figure 3, where the thin solid curve plots $v_{b} \cot \theta_{b n} / v_{g}$ as a function of $\Omega t^{*}$ for typical initial gyrophase angles of each quadrant $\left(\phi_{0}=45^{\circ}, 120^{\circ}, 210^{\circ}, 315^{\circ}\right)$. In each case, the graph starts at $\left(0,-\cos \phi_{0}\right)$ and crosses the $\Omega t^{*}$ axis at $\Omega t_{1}^{*}$ and at $\Omega t_{2}^{*}(=2 \pi)$, as expected.

Next we compare the size of a particle's initial speed normal to the surface of the discontinuity $v_{n}(0)$ and its normal speed at re-encounter $v_{n}\left(t^{*}\right)$. A particle leaving the surface towards upstream must have a positive normal speed $v_{n}(0)>0$ in our framework (see Figure 1) and if the particle reencounters the surface, its normal speed at that moment must be negative, i.e., $v_{n}\left(t^{*}\right)<0$. Therefore, the sum of $v_{n}(0)$ and $v_{n}\left(t^{*}\right)$ will be positive when $v_{n}(0)>\left|v_{n}\left(t^{*}\right)\right|$ and negative when $v_{n}(0)<\left|v_{n}\left(t^{*}\right)\right|$. We will now show that the first case, i.e., the normal speed at re-encounter is smaller than the initial normal speed, occurs when $v_{b}>0$ and that a particle's re-encounter speed is always larger than its initial normal speed whenever $v_{b}<0$. To demonstrate this, let's define the function $F\left(\Omega t^{*}, \phi_{0}\right)$ as the sum of $v_{n}(0)$ given by $(5)$ and $v_{n}\left(t^{*}\right)$ given by $(3)$, normalised by the positive quantity $v_{g} \sin \theta_{b n}$ (see also $(9))$ :

$$
F\left(\Omega t^{*}, \phi_{0}\right) \equiv 2\left[\frac{\sin \phi_{0}-\sin \left(\Omega t^{*}+\phi_{0}\right)}{\Omega t^{*}}\right]+\cos \phi_{0}+\cos \left(\Omega t^{*}+\phi_{0}\right)
$$


(a) $\phi_{0}=45^{\circ}$

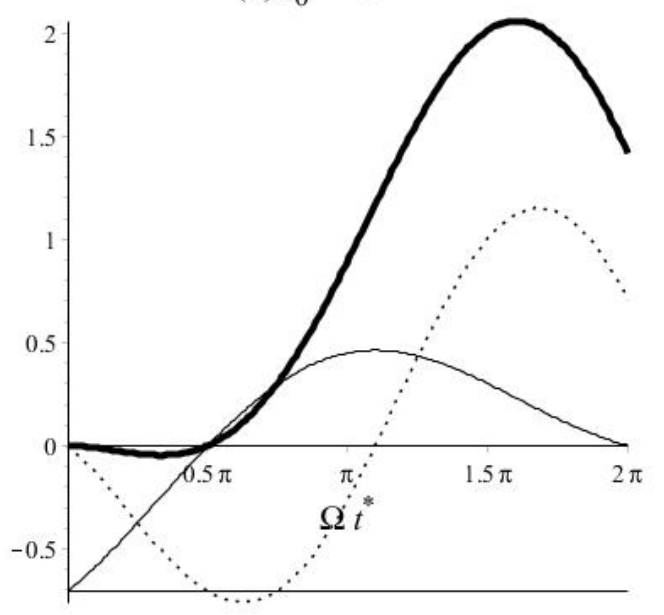

(c) $\phi_{0}=210^{\circ}$

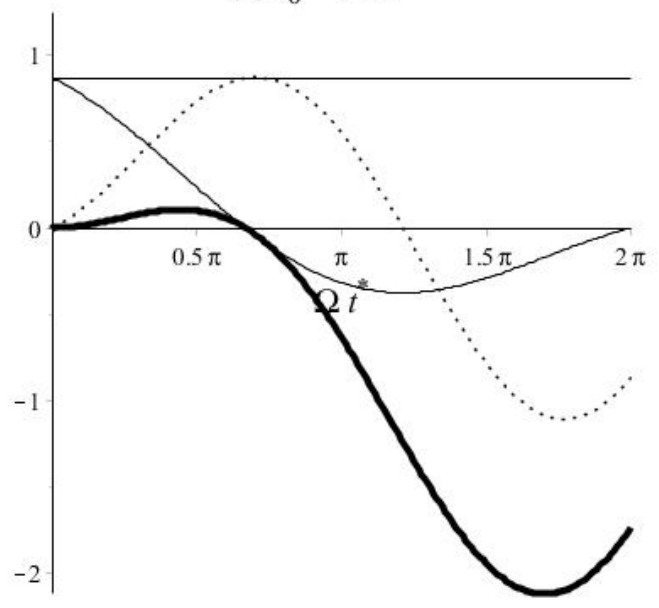

(b) $\phi_{0}=120^{\circ}$

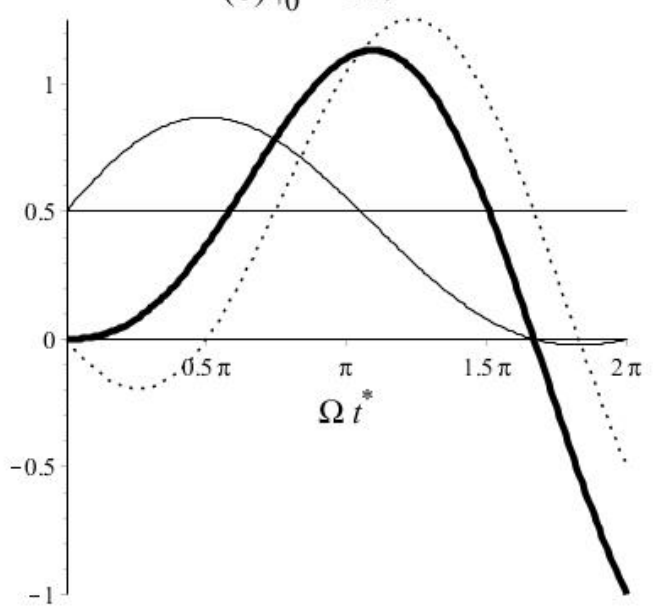

(d) $\phi_{0}=315^{\circ}$

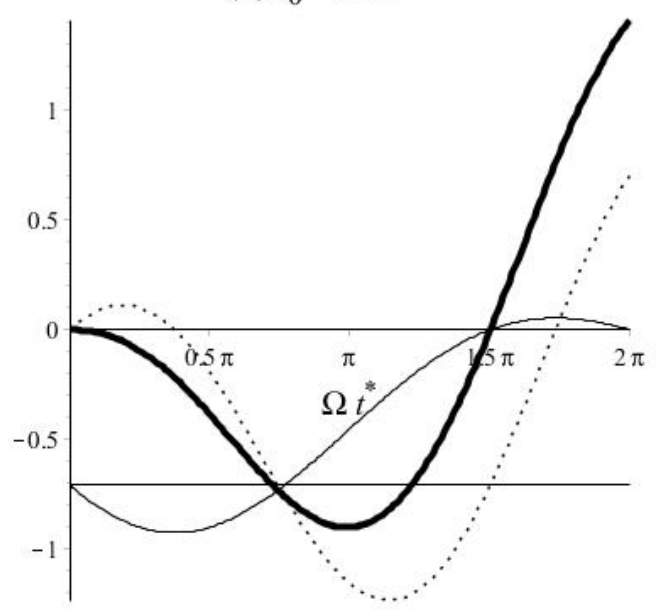

Figure 3: The ratio $\frac{v_{b} \cos \theta_{b n}}{v_{g} \sin \theta_{b n}}$ (thin curve) and the function $F\left(\Omega t^{*}, \phi_{0}\right)$ (see text, thick curve) are plotted together against $\Omega t^{*}$ to illustrate that the two always have the same sign. This indicates that when $v_{b}>0$, the particle's departing normal speed is larger than its normal speed when re-encountering the surface and that the opposite is true when $v_{b}<0$. For reference, the initial normal speed of the particle is pointed upstream only when the thin solid curve lies above the dashed horizontal line at $-\cos \phi_{0}$ and the particle re-encounters the surface from upstream only when the dotted curve is negative (see text for an explanation). 
where (9) has been used to write $v_{b} \cot \theta_{b n} / v_{g}$ in terms of $\phi_{0}$ and $\Omega t^{*}$. Note that within the interval $0 \leq \Omega t^{*}<2 \pi$ we have that $F\left(\Omega t^{*}, \phi_{0}\right)=0$ at $\Omega t^{*}=0$ and at $\Omega t^{*}=\Omega t_{1}^{*}$. Figure 3 shows that the sign of $F\left(\Omega t^{*}, \phi_{0}\right)$, plotted as a thick solid curve, is always the same as that of $v_{b}$ (thin trace), regardless of the value of $\phi_{0}$. As discussed above, this implies that $v_{n}(0)>\left|v_{n}\left(t^{*}\right)\right|$ when $v_{b}>0$ and vice versa.

In order to explain this result analytically we first note that both solid curves in Figure 3, $v_{b} \cot \theta_{b n} / v_{g}$ (thin) and $F\left(\Omega t^{*}, \phi_{0}\right)$ (thick) go through zero at $\Omega t^{*}=\Omega t_{1}^{*}$. It turns out that both $F\left(\Omega t^{*}, \phi_{0}\right)$ and its first derivative with respect to $\Omega t^{*}$ tend to zero as $\Omega t^{*} \rightarrow 0$, i.e., the function $F\left(\Omega t^{*}, \phi_{0}\right)$ has a stationary point at $\Omega t^{*}=0$. Repeated application of L'Hôpital's Rule confirms that the second derivative of $F\left(\Omega t^{*}, \phi_{0}\right)$ with respect to $\Omega t^{*}$ at $\Omega t^{*}=0$ is equal to $\left(-\cos \phi_{0}\right) / 3$. Thus when $\cos \phi_{0}>0$ (figures $3 a, d$ ), $F\left(\Omega t^{*}, \phi_{0}\right)=0$ and also has a local maximum at $\Omega t^{*}=0$. This explains why the solid curve in Figure $3 a, d$ dips to negative values between $0<\Omega t^{*}<$ $\Omega t_{1}^{*}$. The thin curve in Figure 3 is also negative in the same range of $\Omega t^{*}$ when $\cos \phi_{0}>0$ because, as explained earlier, $v_{b} \cot \theta_{b n} / v_{g} \rightarrow-\cos \phi_{0}$ when $\Omega t^{*} \rightarrow 0$. A similar argument applies when $\cos \phi_{0}<0$ (figures $3 b, c$ ).

As noted in the previous section, not all values of $\Omega t^{*}$ (or $\alpha$ ) and $\phi_{0}$ lead to trajectories departing with $v_{n}(0)>0$ or re-encountering the surface on its upstream side (i.e. $v_{n}\left(t^{*}\right)<0$ ). To illustrate where $v_{n}(0)>0$ holds, we have plotted in Figure 3 a dashed horizontal line at a height of $-\cos \phi_{0}$. The initial normal speed is only positive when the thin solid curve that represents $v_{b} \cot \theta_{b n} / v_{g}$ lies above this dashed horizontal line (cf equation (6)). To illustrate where $v_{n}\left(t^{*}\right)<0$, we have plotted $v_{n}\left(t^{*}\right)$ as a dotted curve on Figure 3 . Intervals over which this curve is positive correspond to particles approaching the surface from downstream. Examination of Figure 3c illustrates our previous finding that there can be no particles re-encountering the discontinuity with initial speed pointing upstream and with $180^{\circ}<\phi_{0}<270^{\circ}$.

\section{$5 \quad$ Summary and Discussion}

Previous analytical studies of trajectories in the vicinity of an ideal MHD surface have examined the conditions under which a velocity distribution of ions, initially assumed to be gyrotropic, can become gyrophase-bunched in the specific case when ions reflect off the surface and conserve energy in the process $([20])$. These authors go on to show that a considerable amount of 
structure in velocity space arises from the gyrophase mixing of a gyrophase bunched distribution. Other researchers have focused on alternative generation mechanisms for upstream particles from different (e.g. solar wind or magnetosheath) cold (zero temperature) plasma sources. For example, [39] consider four different potential explanations for ions upstream of Earth's bow shock: magnetic moment-conserving reflection of solar wind ions, specular reflection of solar wind ions, magnetic moment-conserving leakage of magnetosheath ions and leakage of magnetosheath ions parallel to the shock normal. Consistent with the assumption that the plasma is cold, a unique and specific initial pitch angle and gyrophase angle (which may depend on $\theta_{B n}$ and an assumed value for the electrostatic potential rise at the shock) is examined for each of these alternative mechanisms. By contrast, the present theoretical study has examined in detail for the first time the way in which any initial gyrophase and pitch angle of a charged particle affect its ultimate destination after it departs the surface of an ideal planar MHD discontinuity in a steady, homogeneous collisionless plasma flow. We have also determined how the size of the normal speed of a particle that returns to the surface is linked to its initial normal speed. The analysis has been conducted in the HT frame of reference, in which the discontinuity is at rest and the motional electric field is zero. Our main findings may be summarized as follows: a) returning trajectories originate from a very restricted pool of initial gyrophase and pitch angles; b) as $\theta_{b n}$ decreases, the pitch angle range of these returning trajectories reduces; c) the normal speed of any returning particle exceeds its departing normal speed when the particle's guiding centre speed in the HT frame points downstream and vice-versa; d) escaping particles with trajectories that never turn back towards the discontinuity have pitch angles in the range $0^{\circ}<\alpha<\left(90^{\circ}-\theta_{b n}\right)$ [42] but can have any initial gyrophase; e) escaping particles with trajectories temporarily turning back towards the surface originate from a restricted range of pitch angles and this is gyrophase-dependent.

Our theoretical analysis makes it possible to "ring-fence" the portion of initial gyrophase and pitch angle space that would lead to a particle returning to the surface or escaping upstream. For example, particles that return to the surface with normal speed exceeding their initial normal speed must have pitch angles in the band $90^{\circ}<\alpha<\cot ^{-1}\left(-\cos \phi_{0} \tan \theta_{b n}\right)$ which restricts initial gyrophases to lie within either $0^{\circ}<\phi_{0}<90^{\circ}$ or $270^{\circ}<\phi_{0}<360^{\circ}$ (see Figure 2, region A above the horizontal dashed line). Particles escaping the discontinuity could have any initial gyrophase for small pitch angles 
$0<\alpha<90^{\circ}-\theta_{b n}$ (Region $\mathrm{C}$ in Figure 2) or a limited range of gyrophase angles at higher pitch angles (Region $\mathrm{B}$ in Figure 2). In addition, if particles returning to the surface with a normal speed lower than their departing speed remain upstream (consistent with $[6,16]$ ), our analysis suggests that escaping particles pitch angles could extend up to $\alpha=90^{\circ}$ (horizontal dashed line) for $0^{\circ}<\phi_{0}<90^{\circ}$ and $270^{\circ}<\phi_{0}<360^{\circ}$ and up to $\alpha<\cot ^{-1}\left(-\cos \phi_{0} \tan \theta_{b n}\right)$ for $90^{\circ}<\phi_{0}<270^{\circ}$ (see Figure 2 and analysis in Section 3).

The general results above provide an analytical explanation for the way in which initial gyrophase and pitch angle determine the trajectory of any charged particle leaving an ideal MHD discontinuity in a steady flow with constant magnetic field. In practice, the physics of the mechanism(s) responsible for the generation of these particles will ultimately determine the proportions emerging from the surface in each of the gyrophase-pitch angle regions in Figure 2. For example, ions observed upstream of Earth's bow shock may originate from the reflection of solar wind ions or through leakage from the magnetosheath or the magnetosphere. Each of these alternative source mechanisms will constrain the range of possible gyrophases and pitch angles of particles leaving the discontinuity. Previous theoretical studies have assumed a cold plasma source ([39]), so that a unique point in Figure 2 (i.e. a specific $\alpha$ and $\phi_{0}$ ) would be associated with each alternative generation mechanism. As mentioned earlier, if one assumes that all solar wind ions that are specularly reflected at an ideal planar shock have the same incident velocity as the solar wind, these ions depart with $\alpha=2 \theta_{b n}, \phi_{0}=0$ ([39]). Ion beams are typically observed at $\theta_{b n} \sim 50^{\circ}$. In the middle panel in Figure 2, the gyrophase-pitch angle location of these reflected ions would be in the part of region A above the dashed horizontal line, i.e., corresponding to particles that are expected to return to the shock surface with a higher normal speed than when they departed the shock, possibly allowing them to now overcome the electrostatic potential jump at the shock and pass downstream. However, relaxing the assumption of "cold" specular reflection could well place some of the reflected ions in the parts of band A below the horizontal dashed line $\left(\alpha<90^{\circ}\right)$ occupied by particles that return to the shock with a diminished normal speed and that might bounce off again, or even in bands $\mathrm{B}$ or $\mathrm{C}$ occupied by escaping particles. These possibilities are briefly explored below in the context of Earth's bow shock.

In the HT frame, the upstream solar wind travels along the direction of 
the magnetic field with incident bulk velocity:

$$
\mathbf{V}_{i}=-V \frac{\cos \theta_{V n}}{\cos \theta_{b n}} \hat{\mathbf{b}}
$$

where $V$ is the solar wind speed in the frame of an observer at rest on the shock and $\theta_{V n}$ is the acute angle between the incident solar wind velocity direction in the shock rest frame and $\hat{\mathbf{n}}$ (cf. equations (1) and (2) in [39] and Figure 2). In the plasma (solar wind) rest frame most particles will have their own individual peculiar velocity $\mathbf{v}_{\text {pec }}$ with speed $v_{p e c}$. Using the reference axes in Figure 1, the direction of this peculiar velocity can be specified by its angle $\theta_{1}$ relative to $\hat{\mathbf{b}}$ and the angle $\theta_{2}$ relative to the $\hat{\zeta}$ axis of the projection of $\mathbf{v}_{\text {pec }}$ onto the $\hat{\zeta}-\hat{\xi}$ plane (see Figure 1 ). Therefore, in the HT frame, this particle will reach the shock with velocity

$\mathbf{V}_{i}+\mathbf{v}_{p e c}=\left(-V \frac{\cos \theta_{V n}}{\cos \theta_{b n}}+v_{p e c} \cos \theta_{1}\right) \hat{\mathbf{b}}+v_{p e c} \sin \theta_{1} \cos \theta_{2} \hat{\zeta}+v_{p e c} \sin \theta_{1} \sin \theta_{2} \hat{\xi}$

and its incident velocity normal to the shock surface $\mathbf{v}_{\text {in }}$ can be obtained from the dot product of (16) with $\hat{\mathbf{n}}$,

$$
\mathbf{v}_{i n}=\left[\left(-V \frac{\cos \theta_{V n}}{\cos \theta_{b n}}+v_{p e c} \cos \theta_{1}\right) \cos \theta_{b n}+v_{p e c} \sin \theta_{1} \cos \theta_{2} \sin \theta_{b n}\right] \hat{\mathbf{n}}
$$

It has been found that the assumption that ions reflect specularly from the shock accounts reasonably well for the observed velocity distributions immediately upstream and downstream of Earth's bow shock and other features such as the width of the shock's magnetic field "foot" (e.g. [40, 27, 31]). With this simplifying assumption and a little algebra, one can obtain the initial velocity of an ion as it departs the shock after reflection, $\mathbf{v}_{0}$, by subtracting twice the incident normal velocity (17) from (16):

$$
\begin{aligned}
\mathbf{v}_{0}= & {\left[\left(V \frac{\cos \theta_{V n}}{\cos \theta_{b n}}-v_{p e c} \cos \theta_{1}\right) \cos 2 \theta_{b n}-v_{p e c} \sin \theta_{1} \cos \theta_{2} \sin 2 \theta_{b n}\right] \hat{\mathbf{b}}+} \\
& {\left[\left(V \frac{\cos \theta_{V n}}{\cos \theta_{b n}}-v_{p e c} \cos \theta_{1}\right) \sin 2 \theta_{b n}+v_{p e c} \sin \theta_{1} \cos \theta_{2} \cos 2 \theta_{b n}\right] \hat{\zeta}+} \\
& v_{p e c} \sin \theta_{1} \sin \theta_{2} \hat{\xi}
\end{aligned}
$$

Dividing both sides of this expression by the upstream Alfvèn speed $v_{A}$ and noting that $V \cos \theta_{V n} / v_{A}$ is the upstream Mach number $M_{A}$ and that the 
square of the ratio of the ion thermal speed $v_{t h}$ and $v_{A}$ is the ion plasma beta $\beta_{i}[24]$, expression (18) simplifies to:

$$
\begin{aligned}
\frac{\mathbf{v}_{0}}{v_{A}}= & {\left[\left(\frac{M_{A}}{\cos \theta_{b n}}-\frac{v_{p e c}}{v_{t h}} \sqrt{\beta_{i}} \cos \theta_{1}\right) \cos 2 \theta_{b n}-\frac{v_{p e c}}{v_{t h}} \sqrt{\beta_{i}} \sin \theta_{1} \cos \theta_{2} \sin 2 \theta_{b n}\right] \hat{\mathbf{b}}+} \\
& {\left[\left(\frac{M_{A}}{\cos \theta_{b n}}-\frac{v_{p e c}}{v_{t h}} \sqrt{\beta_{i}} \cos \theta_{1}\right) \sin 2 \theta_{b n}+\frac{v_{p e c}}{v_{t h}} \sqrt{\beta_{i}} \sin \theta_{1} \cos \theta_{2} \cos 2 \theta_{b n}\right] \hat{\zeta}+} \\
& \frac{v_{p e c}}{v_{t h}} \sqrt{\beta_{i}} \sin \theta_{1} \sin \theta_{2} \hat{\xi}
\end{aligned}
$$

This expression yields the guiding centre speed $v_{b}$ and allows a simple calculation of the gyrational speed $v_{g}=\sqrt{v_{\zeta}^{2}+v_{\xi}^{2}}$, the initial pitch angle $\alpha$ and gyrophase $\phi_{0}$ of a specularly reflected ion in the HT frame. Note that for a "cold" incoming solar wind $\left(v_{p e c}=0\right)$ expression (19) leads to $\alpha=2 \theta_{b n}, \phi_{0}=0$, as expected. We now analyze the trajectories of three particles with different peculiar velocities $\mathbf{v}_{\text {pec }}$ in the incident solar wind frame. For illustration purposes, we set $M_{A}=2, \theta_{b n}=45^{\circ}, \beta_{i}=1$ and let all three particles share the same speed in the plasma rest frame, $v_{p e c}=2.5 v_{t h}$. This places them in the wings of an incident Maxwellian distribution, where reflected particles seem to originate $([6,7])$. Thus, the only difference between the particles is in the angles $\theta_{1}$ and $\theta_{2}$ that determine the orientation of their peculiar velocity $\mathbf{v}_{\text {pec }}$. Figure 4 plots the displacement normal to the shock as a function of time for the three particles, calculated by substituting the speed components obtained from (19) into the general expression (4) derived in section 2. Particle $1\left(\theta_{1}=40^{\circ}, \theta_{2}=110^{\circ}\right)$ arrives at the shock with normal speed $\mathbf{v}_{\text {in }} \sim-v_{A} \hat{\mathbf{n}}$ which is half of the incident normal bulk speed. It is therefore safe to assume that this particle would not be able to overcome the electrostatic potential jump at the shock and would experience reflection. After reflection, its initial pitch angle $\alpha \sim 73^{\circ}$ and gyrophase $\phi_{0} \sim 59^{\circ}$ lead it to re-encounter the shock. This is a particle in the region labelled $\mathrm{A}$ in Figure 2. Importantly, its pitch angle is below $90^{\circ}$ meaning that it will re-encounter the shock with an even smaller normal speed than at first encounter and presumably bounce again. Particle 2 has $\theta_{1}=30^{\circ}, \theta_{2}=130^{\circ}$ and a normal velocity on arrival at the shock that is again $\mathbf{v}_{\text {in }} \sim-v_{A} \hat{\mathbf{n}}$. This particle's pitch angle and initial gyrophase at the moment it is specularly reflected and departs the shock are $\alpha \approx \phi_{0} \sim 55^{\circ}$. This is a particle in region $\mathrm{B}$, whose trajectory turns towards the shock for a while without ever reaching it, before proceeding to escape upstream (see Figure 4). Finally, particle 3 has $\theta_{1}=20^{\circ}, \theta_{2}=170^{\circ}$ and a normal velocity on arrival at the 
shock $\mathbf{v}_{i n} \sim-0.9 v_{A} \hat{\mathbf{n}}$. Its pitch angle $\alpha \sim 31^{\circ}$ and initial gyrophase $\phi_{0} \sim 17^{\circ}$ after refection place this particle in the region labelled $\mathrm{C}$ in Figure 2. Its trajectory in Figure 4 confirms that this is a particle that escapes upstream without ever re-approaching the shock surface. It is interesting to note that although the 3 particles have identical energy in the solar wind rest frame, their energies in the HT frame are quite different. These energies, $E$, can be calculated in units of the upstream Alfvèn kinetic energy from the squares of the velocity components in (19) or (16):

$$
E=\frac{M_{A}^{2}}{\cos ^{2} \theta_{b n}}+\frac{v_{p e c}^{2}}{v_{t h}^{2}} \beta_{i}-2 \frac{v_{p e c}}{v_{t h}} \sqrt{\beta_{i}} M_{A} \frac{\cos \theta_{1}}{\cos \theta_{b n}}
$$

The angle $\theta_{1}$ between the particle's peculiar velocity $\mathbf{v}_{p e c}$ and $\hat{\mathbf{b}}$ is entirely responsible for the difference in energy in the HT frame between these three particles. Thus, Particle 2 (region B) is approximately 2.1 times as energetic as Particle 3 (region C), while Particle 1 has more than 3.5 times the energy of Particle 3. This simple example illustrates how one may obtain higher pitch angle escaping particles (region B) that are consideraby more energetic than lower pitch angle particles (region $\mathrm{C}$ ) even though the particles were initially close to each other in the incident distribution. A more systematic study of how a distribution of particles interacts in this way with a shock may lead to an understanding of some of the, as yet, unexplained features of beams observed upstream of Earth's bow shock, namely, the high enery tails at high pitch angles in their distributions ([32]), their distinctive parallel and perpendicular temperature profiles ([29]) or the two populations distinguishable by their pitch angle in the numerical study by [17].

High-resolution measurements by the CLUSTER spacecraft indicate that field-aligned beams share a common origin with the reflected-gyrating ions at Earth's bow shock [34, 25] and this is consistent with test particle calculations which found that backstreaming ions can be produceded by increasing the pitch angle of some of the incident ions [6]. A process of pitch angle scattering by low frequency waves has been invoked to explain the selection of the beam ions out of the reflected-gyrating non-gyrotropic distribution $[34,25]$. Our results confirm earlier findings of numerical calculations [6] suggesting that such mechanism might not be necessary and that the two populations might arise naturally when allowing for a range of initial gyrophase and pitch angles in the incident distribution. The precise fraction of reflecting particles in regions $\mathrm{A}, \mathrm{B}$ or $\mathrm{C}$ and their respective velocity ranges 


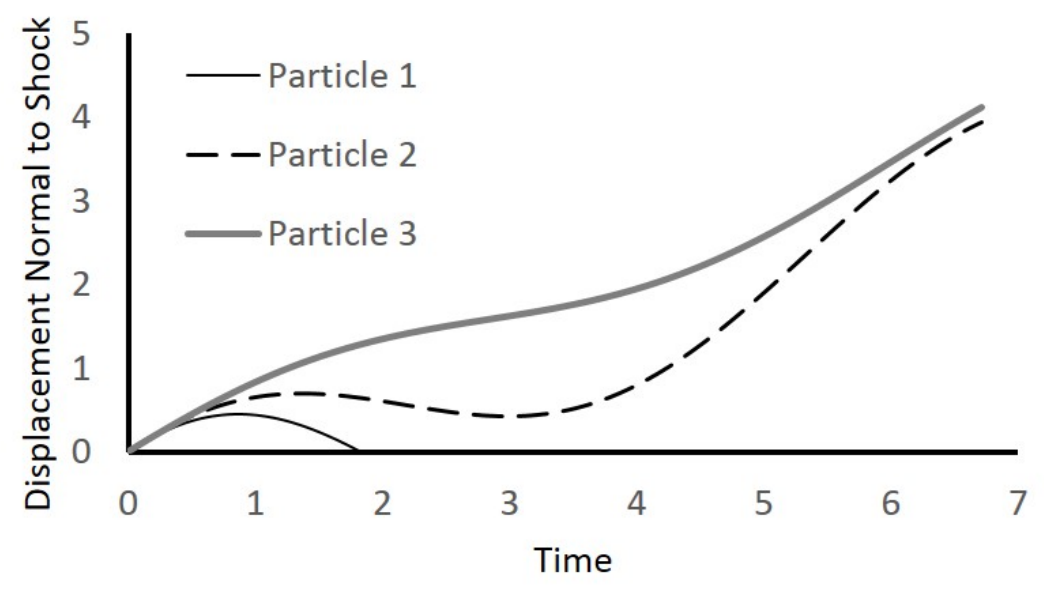

Figure 4: Displacement normal to an ideal shock surface, in units of $v_{A} / \Omega$, as a function of time, in units of $\Omega^{-1}$, of three particles specularly reflected when the upstream Mach number $M_{A}=2$, the ion plasma beta $\beta_{i}=1$ and $\theta_{b n}=45^{\circ}$. All three particles have a speed in the incident plasma rest frame of 2.5 thermal speeds and orientations (see text) given by $\theta_{1}=40^{\circ}, \theta_{2}=110^{\circ}$ (Particle 1), $\theta_{1}=30^{\circ}, \theta_{2}=130^{\circ}$ (Particle 2) and $\theta_{1}=20^{\circ}, \theta_{2}=170^{\circ}$ (Particle $3)$. 
and temperatures will depend sensitively on the properties of the incident solar wind distribution, in particular, its high-speed wing, and on the orientation of the upstream magnetic field. An analytical investigation of the properties of ion populations at Earth's bow shock generated by assuming specular reflection of an incident solar wind with a distribution of velocities is beyond the scope of the present paper. Such investigation could be conducted using the framework and general results we have presented here. We suggest that a study of the details of the incident solar wind velocity distributions in the CLUSTER data and of the associated escaping beams may reveal significant links between the two. It would also be interesting to study the resulting range of initial pitch angles and gyrophases if the solar wind distribution is better fitted by a nonthermal kappa distribution (e.g. [37]) than a Maxwellian. The identification of the gyrophase-pitch angle structure of returning particles might also provide valuable input to studies of the dominant instabilities responsible for the collisionless thermalization of the ion distributions downstream of collisionless shocks.

In common with many previous studies (e.g. [39, 14, 35]), we have modelled the discontinuity surface as an ideal infinite plane in a steady homogeneous flow. We have therefore neglected a host of physical phenomena that could significantly affect particle trajectories, such as any non-stationarity and curvature of the surface, its structure and finite width and the effects of waves and turbulence. Nevertheless, we hope that our analysis of the fundamental equations of motion for charged particles will contribute to a better understanding of the underlying role of initial gyrophase and pitch angle in a particle's subsequent evolution near the surface of an MHD discontinuity.

Acknowledgements I am grateful to Alison Bruce, Steve Ellacott and Steve Schwartz for their comments on an early version of this manuscript and to both referees for their helpful suggestions.

\section{References}

[1] V. A. Artemyev, V. Angelopoulos, I. Y. Vasko, A. Runov, L. A. Avanov, B. L. Giles, C. T. Russell, and R. J. Strangeway. On the Kinetic Nature of Solar Wind Discontinuities. Geophysical Research Letters, 46(3):11851194, Feb 192019. 
[2] S. D. Bale, M. A. Balikhin, T. S. Horbury, V. V. Krasnoselskikh, H. Kucharek, E. Möbius, S. N. Walker, A. Balogh, D. Burgess, B. Lembège, E. A. Lucek, M. Scholer, S. J. Schwartz, and M. F. Thomsen. Quasi-perpendicular Shock Structure and Processes. Space Science Reviews, 118:161-203, June 2005.

[3] A. L. Brinca. A first assessment of the free energy in nongyrotropic plasmas. Journal of Atmospheric and Solar-Terrestrial Physics, 62:701709, May 2000.

[4] D. Burgess. Collisionless shocks. In Introduction to Space Physics, pages 129-163. Cambridge University Press, Cambridge CB2 1RP, UK, 1995.

[5] D. Burgess, E. Möbius, and M. Scholer. Ion Acceleration at the Earth's Bow Shock. Space Science Reviews, 173:5-47, November 2012.

[6] D. Burgess and S. J. Schwartz. The dynamics and upstream distributions of ions reflected at the earth's bow shock. Journal of Geophysical Research, 89:7407-7422, September 1984.

[7] D. Burgess, W. P. Wilkinson, and S. J. Schwartz. Ion distributions and thermalization at perpendicular and quasi-perpendicular supercritical collisionless shocks. Journal of Geophysical Research, 94:8783-8792, July 1989 .

[8] P. D. Convery, D. Schriver, M. Ashour-Abdalla, and R. L. Richard. Wave-particle interactions associated with nongyrotropic distribution functions: A hybrid simulation study. Journal of Geophysical Research (Space Physics), 107:1013, January 2002.

[9] F. de Hoffmann and E. Teller. Magneto-Hydrodynamic Shocks. Physical Review, 80:692-703, November 1950.

[10] S. A. Fuselier and M. F. Thomsen. He-2+ in field-aligned beams - ISEE results. Geophysical Research Letters, 19(5):437-440, March 1992.

[11] S. A. Fuselier, M. F. Thomsen, S. P. Gary, S. J. Bame, C. T. Russell, and G. K. Parks. The Phase relationship between Gyrophase-bunched Ions and MHD-like Waves. Geophysical Research Letters, 13(1):60-63, JAN 1986. 
[12] S. P. Gary. Electromagnetic Ion Ion Instabilities and Their Consequences in Space Plasmas - A Review. Space Science Reviews, 56(3-4):373-415, May 1991.

[13] S. P. Gary, B. J. Anderson, R. E. Denton, S. A. Fuselier, M. E. McKean, and D. Winske. Ion anisotropies in the magnetosheath. Geophysical Research Letters, 20:1767-1770, September 1993.

[14] M Gedalin. Transmitted ions and ion heating in nearly perpendicular low-Mach number shocks. Journal of Geophysical Research: Space Physics, 101(A7):15569-15578, Jul 11996.

[15] M. Gedalin. Collisionless relaxation of non-gyrotropic downstream ion distributions: dependence on shock parameters. Journal of Plasma Physics, 81(6), Dec 2015.

[16] M. Gedalin. Transmitted, reflected, quasi-reflected, and multiply reflected ions in low-Mach number shocks. Journal of Geophysical Research: Space Physics, 121(11):10754-10767, November 2016.

[17] M. Gedalin, M. Liverts, and M. A. Balikhin. Distribution of escaping ions produced by non-specular reflection at the stationary quasiperpendicular shock front. Journal of Geophysical Research: Space Physics, 113(A5), 2008.

[18] J. T. Gosling and A. E. Robson. Ion reflection, gyration, and dissipation at supercritical shocks. In B. T. Tsurutani and R. G. Stone, editors, Collisionless Shocks in the Heliosphere: Reviews of Current Research, Geophysical Monograph Series, pages 141-152. AGU, Washington, D. C., 1985 .

[19] Zhifang Guo, Yu Lin, Xueyi Wang, and Aimin Du. Magnetosheath Reconnection Before Magnetopause Reconnection Driven by Interplanetary Tangential Discontinuity: A Three-Dimensional Global Hybrid Simulation With Oblique Interplanetary Magnetic Field. Journal of Geophysical research: Space Physics, 123(11):9169-9186, Nov 2018.

[20] C. Gurgiolo, G. K. Parks, and B. H. Mauk. Upstream gyrophase bunched ions: A mechanism for creation at the bow shook and the growth of velocity space structure through gyrophase mixing. Journal of Geophysical Research, 88:9093-9100, November 1983. 
[21] C. Gurgiolo, G. K. Parks, B. H. Mauk, C. S. Lin, K. A. Anderson, R. P. Lin, and H. Reme. Non-E x B Ordered Ion-Beams Upstream of the Earths Bow Shock. Journal of Geophysical Research (Space Physics), 86(NA6):4415-4424, 1981.

[22] M. Hoshino and T. Terasawa. Numerical Study of the Upstream Wave Excitation Mechanism. 1. Nonlinear Phase Bunching of Beam Ions. Journal of Geophysical Research (Space Physics), 90(NA1):57-64, 1985.

[23] F. M. Ipavich, G. Gloeckler, D. C. Hamilton, L. M. Kistler, and J. T. Gosling. Protons and alpha-particles in field-aligned beams upstream of the bow shock. Geophysical Research Letters, 15(10):1153-1156, September 1988 .

[24] M. G. Kivelson. Physics of space plasmas. In Introduction to Space Physics, pages 27-57. Cambridge University Press, Cambridge CB2 1RP, UK, 1995.

[25] H. Kucharek, E. Möbius, M. Scholer, C. Mouikis, L. Kistler, T. Horbury, A. Balogh, H. Rème, and J. Bosqued. On the origin of field-aligned beams at the quasi-perpendicular bow shock: multi-spacecraft observations by Cluster. Annales Geophysicae, 22:2301-2308, July 2004.

[26] Z. Liu, D. L. Turner, V. Angelopoulos, and N. Omidi. THEMIS observations of tangential discontinuity-driven foreshock bubbles. Geophysical Research Letters, 42(19):7860-7866, Oct 162015.

[27] W. A. Livesey, C. T. Russell, and C. F. Kennel. A Comparison of Specularly Reflected Gyrating Ion Orbits with Observed Shock Foot Thicknesses. Journal of Geophysical Research-Space Physics, 89(NA8):68246828, 1984.

[28] C. Mazelle, K. Meziane, D. LeQueau, M. Wilber, J. P. Eastwood, H. Rème, J. A. Sauvaud, J. M. Bosqued, I. Dandouras, M. McCarthy, L M. Kistler, B. Klecker, A. Korth, M. B. Bavassano-Cattaneo, G. Pallocchia, R. Lundin, and A. Balogh. Production of gyrating ions from nonlinear wave-particle interaction upstream from the Earth's bow shock: A case study from Cluster-CIS. Planetary and Space Science, 51(12):785-795, Oct 2003. 
[29] K. Meziane, A. M. Hamza, M. Wilber, C. Mazelle, and M. A. Lee. On the Field-Aligned Beam Thermal Energy. Journal of Geophysical Research (Space Physics), 118(11):6946-6954, Nov 2013.

[30] K. Meziane, C. Mazelle, R. P. Lin, D. LeQueau, D. E. Larson, G. K. Parks, and R. P. Lepping. Three-dimensional observations of gyrating ion distributions far upstream from the Earth's bow shock and their association with low-frequency waves. Journal of Geophysical Research (Space Physics), 106(A4):5731-5742, Apr 12001.

[31] K. Meziane, C. Mazelle, M. Wilber, D. LeQueau, J. P. Eastwood, H. Rème, I. Dandouras, J. A. Sauvaud, J. M. Bosqued, G. K. Parks, L. M. Kistler, M. McCarthy, B. Klecker, A. Korth, M. B. BavassanoCattaneo, R. Lundin, and A. Balogh. Bow shock specularly reflected ions in the presence of low-frequency electromagnetic waves: a case study. Annales Geophysicae, 22(7):2325-2335, 2004. Meeting on SpatioTemporal Analysis and Multipoint Measurements in Space, Orleans, France, May 12-16, 2003.

[32] K. Meziane, M. Wilber, A. M. Hamza, C. Mazelle, G. K. Parks, H. Rème, and E. A. Lucek. Evidence for a high-energy tail associated with foreshock field-aligned beams. Journal of Geophysical Research (Space Physics), 112:A01101, January 2007.

[33] K. Meziane, M. Wilber, C. Mazelle, D. Le Quéau, H. Kucharek, E. A. Lucek, H. Rème, A. M. Hamza, J. A. Sauvaud, J. M. Bosqued, I. Dandouras, G. K. Parks, M. McCarthy, B. Klecker, A. Korth, M. B. Bavassano-Cattaneo, and R. N. Lundin. Simultaneous observations of field-aligned beams and gyrating ions in the terrestrial foreshock. Journal of Geophysical Research (Space Physics), 109:A05107, May 2004.

[34] E. Möbius, H. Kucharek, C. Mouikis, E. Georgescu, L. M. Kistler, M. A. Popecki, M. Scholer, J. M. Bosqued, H. Rème, C. W. Carlson, B. Klecker, A. Korth, G. K. Parks, J. C. Sauvaud, H. Balsiger, M.-B. Bavassano-Cattaneo, I. Dandouras, A. M. Dilellis, L. Eliasson, V. Formisano, T. Horbury, W. Lennartsson, R. Lundin, M. McCarthy, J. P. McFadden, and G. Paschmann. Observations of the spatial and temporal structure of field-aligned beam and gyrating ring distributions at the quasi-perpendicular bow shock with Cluster CIS. Annales Geophysicae, 19:1411-1420, October 2001. 
[35] L. Ofman, M. Balikhin, C. T. Russell, and M. Gedalin. Collisionless relaxation of ion distributions downstream of laminar quasi-perpendicular shocks. Journal of Geophysical Research- Space Physics, 114, Sep 30 2009 .

[36] M. Oka, T. Terasawa, Y. Saito, and T. Mukai. Field-aligned beam observations at the quasi-perpendicular bow shock: Generation and shock angle dependence. Journal of Geophysical Research: Space Physics, 110(A5), May 142005.

[37] G. Parks. Physics Of Space Plasmas: An Introduction, Second Edition. Westview Press, Oxford OX2 9JJ, UK, 2004.

[38] M. S. Ruderman, E. Vickers, I. Ballai, and R. Erdelyi. Propagation of leaky surface waves on contact magnetohydrodynamic discontinuities in incompressible plasmas. Physics of Plasmas, 25(12), Dec 2018.

[39] S. J. Schwartz, M. F. Thomsen, and J. T. Gosling. Ions upstream of the earth's bow shock - A theoretical comparison of alternative source populations. Journal of Geophysical Research, 88:2039-2047, March 1983.

[40] N. Sckopke, G. Paschmann, S. J. Bame, J. T. Gosling, and C. T. Russell. Evolution of ion distributions across the nearly perpendicular bow shock - Specularly and non-specularly reflected-gyrating ions. Journal of Geophysical Research, 88:6121-6136, August 1983.

[41] N. Sckopke, G. Paschmann, A. L. Brinca, C. W. Carlson, and H. Lühr. Ion thermalization in quasi-perpendicular shocks involving reflected ions. Journal of Geophysical Research, 95:6337-6352, May 1990.

[42] M. F. Thomsen. Upstream suprathermal ions. In B. T. Tsurutani and R. G. Stone, editors, Collisionless Shocks in the Heliosphere: Reviews of Current Research, Geophysical Monograph Series, pages 253-270. AGU, Washington, D. C., 1985.

[43] G. Voitcu and M. Echim. Tangential deflection and formation of counterstreaming flows at the impact of a plasma jet on a tangential discontinuity. Geophysical Research Letters, 44(12):5920-5927, Jun 282017. 